\title{
Efecto neuroprotector de los cannabinoides en las enfermedades neurodegenerativas
}

\author{
Neuroprotective Effect of Cannabinoids in Neurodegenerative Diseases \\ Carlos Suero-García · Lucia Martín-Banderas · Mª Ángeles Holgado \\ Departamento de Farmacia y Tecnología Farmacéutica. Facultad de Farmacia. Universidad de Sevilla
}

\section{Artículo de Revisión Review Article}

\section{Correspondencia} Correspondence

$\mathrm{M}^{\mathrm{a}}$ Ángeles Holgado

Departamento de Farmacia y Tecnología Farmacéutica. Facultad de Farmacia.

Universidad de Sevilla. c/ Profesor García González, nº 2, 41012 Sevilla holgado@us.es

Financiación

Fundings

Trabajo realizado sin financiación.

Conflicto de interés Competing interest

Los autores declaran que no existe conflicto de interés.

Received: 02.02.2015 Accepted: 15.03.2015

\section{RESUMEN}

Objetivos. Se analiza la situación actual de las investigaciones relacionadas con las sustancias cannabinoides, así como su interacción con el organismo, clasificación, efectos terapéuticos y su uso en las enfermedades neurodegenerativas.

Métodos. Se realiza una exhaustiva revisión bibliográfica relacionada con las sustancias cannabinoides y sus derivados sintéticos, haciendo especial hincapié en la forma de interactuar con el organismo y los efectos que provocan dichas interacciones. Concretamente, se estudiarán sus efectos neuroantiinflamatorio y analgésico lo que conlleva al efecto neuroprotector en enfermedades neurodegenerativas tales como Alzheimer, Parkinson, Huntington, esclerosis múltiple y esclerosis lateral amiotrófica.

Resultados. Desde hace miles de años la planta Cannabis Sativa ha sido utilizada por muchas culturas con distintos fines, de ocio, textiles, analgésicos, pero no es hasta finales del siglo XX cuando se empieza a incentivar los estudios científicos relacionados con ésta. La planta posee una mezcla de unos 400 componentes, de los cuales 60 pertenecen al grupo de los cannabinoides siendo los principales el cannabinol, cannabidiol y tetrahidrocannabinol. Con el descubrimiento de las sustancias cannabinoides, sus derivados, y los receptores que interactúan, se amplían las posibilidades terapéuticas teniendo un especial interés el efecto neuroprotector que estas sustancias contienen. Conclusiones. Se ha demostrado el gran potencial de los cannabinoides como sustancias terapéuticas más allá de su uso analgésico o antiemético, esto es, en enfermedades neurodegenerativas en las que pueden no solo disminuir los síntomas, sino frenar el proceso de la enfermedad. Otra posible aplicación puede ser en el campo oncológico, siendo particularmente intensa la actividad investigadora realizada en los últimos 15 años.

Palabras clave: Cannabis sativa, cannabinoides, neuroprotección, neurodegeneración, cáncer

\section{ABSTRACT}

Objectives. It is analysed the actual situation of the investigations related to cannabinoids substances, as well as their interaction with the organism, classification, therapeutics effects and their use in neurodegenerative diseases.

Methods. This study is based on the review of multiples scientific articles directly related with the cannabinoides substance and its synthetic derivates, with a special attention on the way the organism interacts and the effects the substances cause in it. Specifically, the study will get deep into the neuroantiinflammatory and analgesic effects of these substances, related with the neuroprotector effect in neurodegenerative diseases such as Alzheimer, Parkinson, Multiple Sclerosis and Amyotrophic Lateral Sclerosis.

Results. From thousands years, the Cannabis Sativa has been used by multiples cultures with different purposes such as joy, textile, analgesics etc. But is not till ends of the $X X$ century when there are diverse scientific studies related with the Cannabis encouraged. The plant has 400 components, 60 of them belong to the cannabinoides group. The main ones are cannabinol, cannabidiol and tetrahidrocannabinol. With the discoverment of the cannabinoids substances, its derivates, and the receptors which interact 
with them, it is increased the therapeutic possibilities and remarking the neuroprotective effect which these substances contain.

Conclusions. It has been demostraded the huge potential of the cannabinoids as therapeutic substances apart from its analgesic and antiemetic uses, that is, neurodegenaritve diseases in which they can not only decrease its symptons but stop disease process. Another possible application could be in the oncologic area, being particularly intense the investigation's activity realised the last 15 years.

Keywords: Cannabis Sativa, cannabinoids, neuroprotection, neurodegeneration, cancer.

\section{INTRODUCCIÓN}

La planta Cannabis Sativa, también conocida como cáñamo indiano, ha sido utilizada desde hace más de 8000 años, expandiéndose su uso por todo el mundo. Dicha difusión fue causada por sus usos analgésicos, anestésicos, recreativos e incluso textiles ${ }^{1}$.

Es una planta herbácea, cuyo interés farmacológico reside en los cannabinoides, presentes en las sumidades floridas y en la resina de las plantas. La planta posee una mezcla de unos 400 componentes, de los cuales 60 pertenecen al grupo de los cannabinoides. Los principales son cannabinol (CBN), cannabidiol (CBD) y tetrahidrocannabinol (THC), siendo este último al que se le atribuyen las propiedades psicoactivas ${ }^{2}$.
El objetivo del presente trabajo es analizar la situación actual de las investigaciones relacionadas con las sustancias cannabinoides, así como su interacción con el organismo, clasificación, efectos terapéuticos y su uso en las enfermedades neurodegenerativas.

\section{MATERIAL Y MÉTODOS}

Para la realización de este trabajo se realizó, inicialmente, un estudio de los tratados clásicos sobre la materia, completándose con una búsqueda bibliográfica en las bases de datos Pubmed, Scopus, catálogo Fama de la universidad de Sevilla y Science Direct. Esta última información obtenida se filtró teniendo en cuenta sobre todo dos características fundamentales: estudios publicados en los últimos cinco años y estudios en los que las palabras clave apareciesen en el título y/o resumen. Se consideraron válidos todos aquellos artículos que, tras su minuciosa lectura, servían al propósito general de este trabajo.

\section{RESULTADOS Y DISCUSIÓN}

A pesar de que el conocimiento del cannabis está extendido por todo el mundo, en el presente artículo se profundizará en los siguientes puntos: (i) interacción en el organismo, (ii) clasificación de los cannabinoides y (iii) descripción de los efectos que provoca.

Figura 1. Representación esquemática de los receptores CB1 y CB2. Contienen siete segmentos transmembrana; Los aminoácidos sombreados son comunes mientras que los blancos son diferentes. $\Psi$ : sitios de glicosilación; e1, e2, e3: asas extracelulares; i1, i2, i3: asas intracelulares.

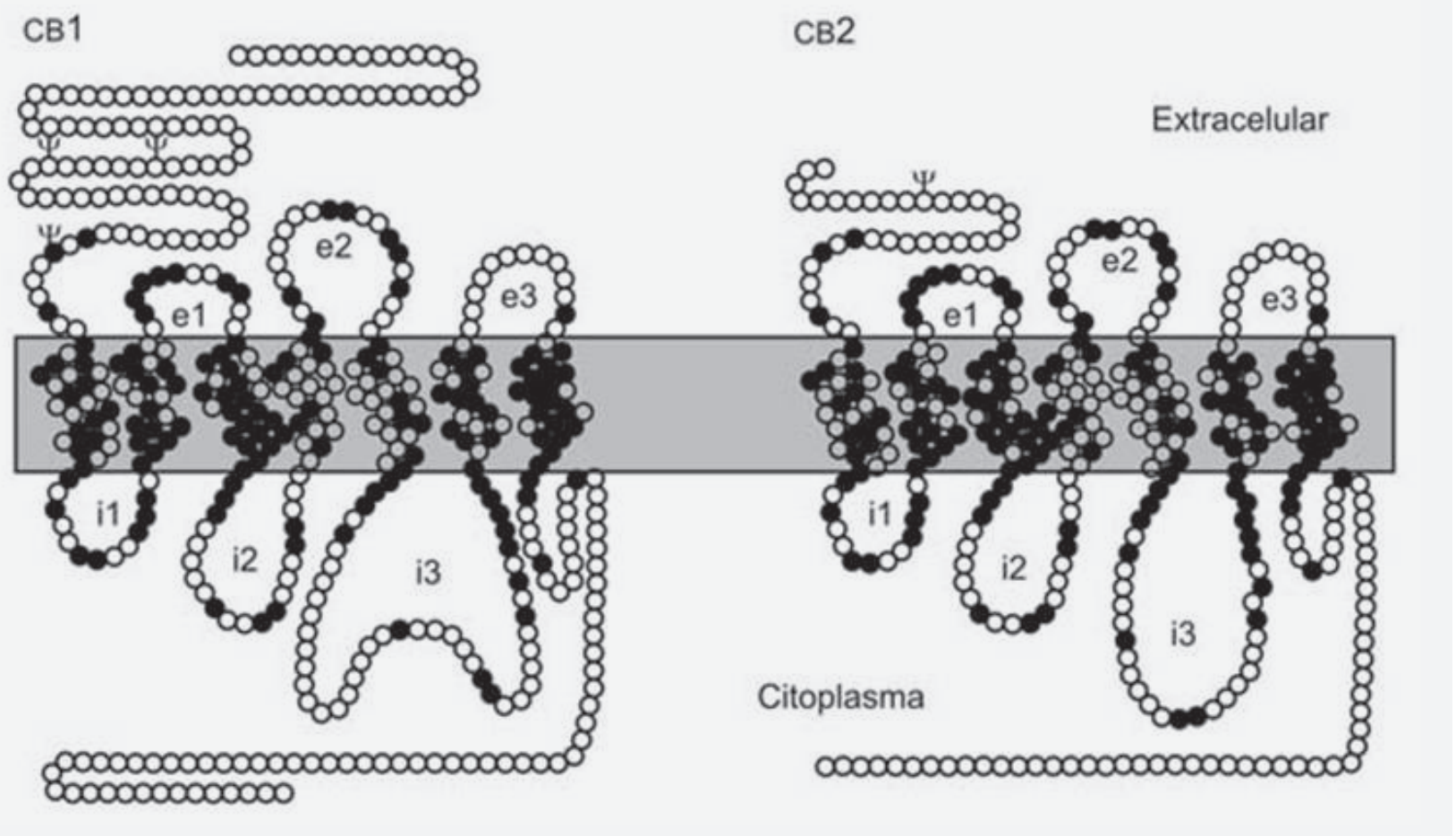




\section{Receptores cannabinoides}

La actividad de estas sustancias se debe a la interacción de las mismas con unos receptos unidos a proteína G llamados receptores cannabinoides.

Hasta la fecha, se han identificado mediante clonación molecular en ratas, dos tipos de receptores (Figura 1). Los receptores CB1 son los más abundantes entre los receptores unidos a proteína $\mathrm{G}$ en el sistema nervioso central de mamíferos (SNC). Están presentes en niveles particularmente altos en el cerebelo, hipocampo y los ganglios basales, donde median la inhibición de la liberación de varios neurotransmisores excitadores e inhibitorios. En menor cantidad también están presentes en el sistema nervioso periférico, así como en las células somáticas de la mayoría de los tejidos.

Se ha confirmado que el receptor CB2 tiene una acción diferente sobre el organismo al que presenta el CB1. Se expresan predominantemente, aunque no exclusivamente, en las células inmunes y hematopoyéticas. Más recientemente, receptores CB2 funcionales se han identificado tanto en neuronas y células gliales del $\mathrm{SNC}^{3}$.

Tanto los receptores CB1 como CB2, a través de proteínas $\mathrm{G}$, inhiben la adenilciclasa y regulan los canales de iones, incluyendo los canales de potasio acoplados a proteínas $\mathrm{G} o$ los canales de calcio dependientes de voltaje tipo N. Los receptores cannabinoides regulan, de manera independiente a las proteínas $G$, la actividad de una variedad de quinasas intracelulares. Afectan también a la acción de varios neurotransmisores como acetilcolina, dopamina, GABA, glutamato, serotonina, norepinefrina, y opioides endógenos ${ }^{4}$.

\section{Clasificación de las sustancias cannabinoides}

Según su origen, se reconocen tres tipos generales de cannabinoides: los fitocannabinoides sintetizados de forma natural por la planta del cannabis; los cannabinoides endógenos o endocannabinoides, producidos de forma natural por animales y humanos; y los cannabinoides sintéticos, compuestos similares generados en el laboratorio ${ }^{5}$. La estructura principal fitocannabinoide fue identificada como un anillo tricíclico constituido a partir de un anillo de fenol, un anillo de pirano central, y un anillo de ciclohexilo monoinsaturado. Se compone de 21 carbonos en total $^{6}$ (Figura 2).

El cannabidiol (CBD) es un compuesto bicíclico al estar el anillo de tetrahidropirano escindido por lo que está prácticamente desprovisto de propiedades psicoactivas ${ }^{7}$ (Figura 3).

La sustancia con mayor potencia en la actividad es el delta-9-tetrahidrocannabinol $\left(\Delta^{9}-\mathrm{THC}\right)$, siendo el cannabinol
(CBN) un derivado del $\Delta^{9}$-THC, producto de su degradación y 10 veces menos potente ${ }^{5}$. A pesar de que el CBD no es una sustancia con propiedades psicoactivas, estudios recientes sugieren un amplio campo de posibles efectos terapéuticos en numerosas enfermedades ${ }^{8}$ además de ser capaz de antagonizar el efecto del $\Delta^{9}-\mathrm{THC}^{9}$. Figura 2. Estructura química del delta-9-tetrahidrocannabinol
o $\triangle 9$-THC

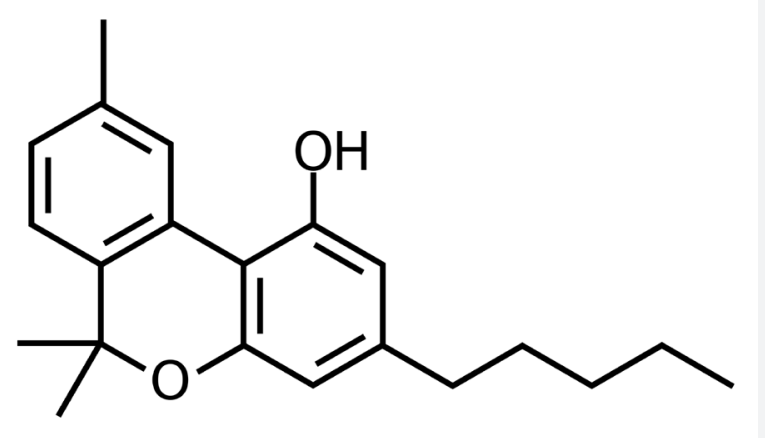

Figura 3. Estructura química del cannabidiol o CBD

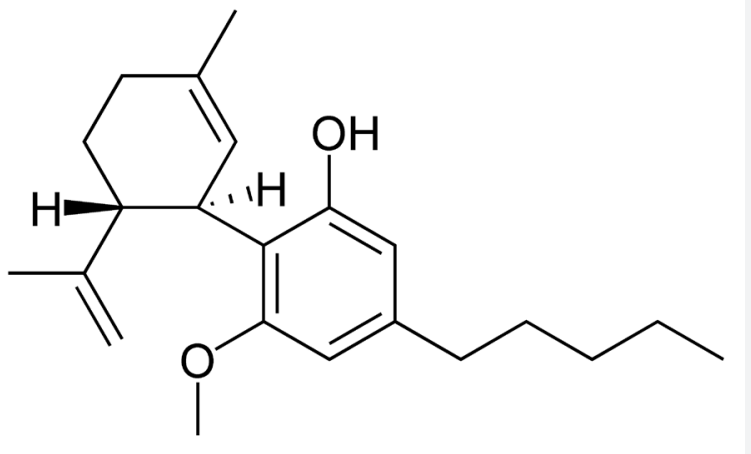

El segundo grupo son los cannabinoides endógenos o endocannabinoides. El primero identificado fue una amida lipídica aislada del cerebro porcino, la etanolamida $\mathrm{N}$-araquidonoil (anandamida o AEA). Estudios posteriores han revelado que la AEA se genera in vivo a partir de precursores de fosfolípidos de membrana. Un segundo endocannabinoide, aislado 3 años más tarde en el intestino y cerebro, era un éster de glicerol, 2-araquidonoilglicerol (2-AG), que se metaboliza preferentemente por la monoacilglicerol lipasa, con la participación adicional de la AB-hidrolasa. Otros ligandos cannabinoides endógenos identificados incluyen amidas, (dopamina $\mathrm{N}$-araquidonoil), ésteres (virodamina y N-dihomo-g-linolenoiletanolamina), y algunos éteres, (noladin-éter) ${ }^{3}$ (Figura 4).

El tercer grupo son los cannabinoides sintéticos derivados del $\Delta^{9}$-THC, como dronabinol y nabilona, usados por sus características antieméticas. Se han desarrollado una gran variedad de ellos como HU210, con una alta afinidad por el 
receptor CB1; CP55940, que aumenta la potencia terapéutica del $\Delta^{9}$-THC o WIN55212 (Figura 5).

Hasta ahora todos los cannabinoides citados producen un efecto agonista. En los últimos años se ha descubierto y desarrollado cannabinoides sintéticos antagonistas como es el SR141716A o rimonabant (Figura 6), que antagoniza el efecto de la anandamida con una gran afinidad por los receptores CB1. Estos cannabinoides antagonistas no tienen un efecto contrario a los agonistas, ya que se ha descubierto que aparte de estos dos receptores intervienen otros no unidos a proteína $G$, aunque sí que tienen actividad anticannabimimética9.

Efectos en el organismo de las sustancias cannabinoides

El $\Delta^{9}$-THC y sus derivados sintéticos alteran el equilibrio natural de los endocannabinoides dando a lugar a una serie de respuestas en el organismo.

A nivel cognitivo, se ha demostrado que provoca una disminución en la coordinación motora, el procesamiento de las operaciones temporales y el aprendizaje asociativo. También provoca alteraciones en la percepción cognitiva y auditiva. Esto es debido a la presencia de receptores en el cerebelo y la consecuente alteración de sus funciones ${ }^{7}$.

A nivel psicológico, existen numerosos estudios contradictorios, ya que algunos afirman que el consumo crónico puede aumentar el riesgo y disminuir la edad de padecer un brote psicótico y aumentar la ansiedad, mientras que otros lo desmienten ${ }^{4}$.

A nivel inmunológico, estudios recientes en ratas y ratones afirman el hecho de que el $\Delta^{9}$-THC y CBD tienen un efecto anticarcinogénico. El mecanismo de acción antitumoral se basa en la capacidad de estos compuestos para: (i) promover la muerte apoptótica de las células cancerosas, (ii) inhibir la angiogénesis del tumor y (iii) reducir la migración de las células cancerosas. Se ha propuesto que la apoptosis inducida por éste se basa en un aumento de la producción de especies reactivas de oxígeno, un mecanismo que parece funcionar también en células de glioma ${ }^{10}$.

También se están realizando estudios sobre el SIDA, en los que hay evidencias que demuestran que el uso de sustancias cannabinoides pueden mejorar la capacidad del huésped para controlar la carga viral y prolongar la superviven$\mathrm{cia}^{11}$.

Estas sustancias también causan efectos cardiovasculares, como taquicardia, aumento de la presión arterial y, con dosis más altas, hipotensión, bradicardia y vasodilatación de las arterias coronarias.
Figura 4. Estructura química de un endocannabinoide, anandamida o AEA

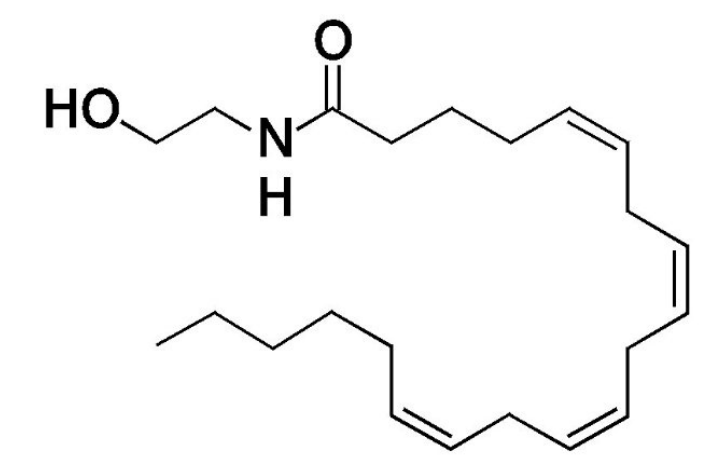

Figura 5. Estructura química de un cannabinoide sintético WIN55212

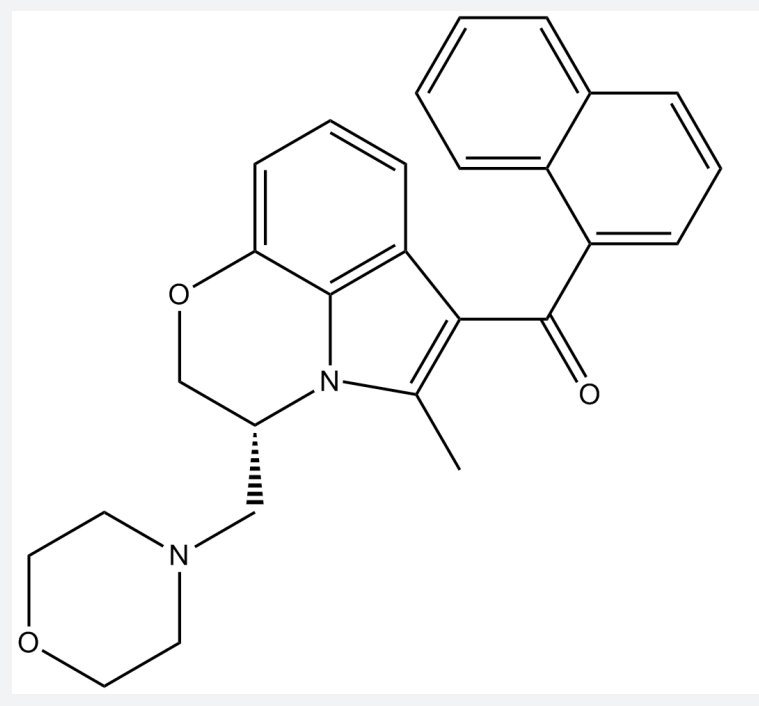

Figura 6. Estructura química del SR141716A o rimonabant, principal cannabinoide antagonista

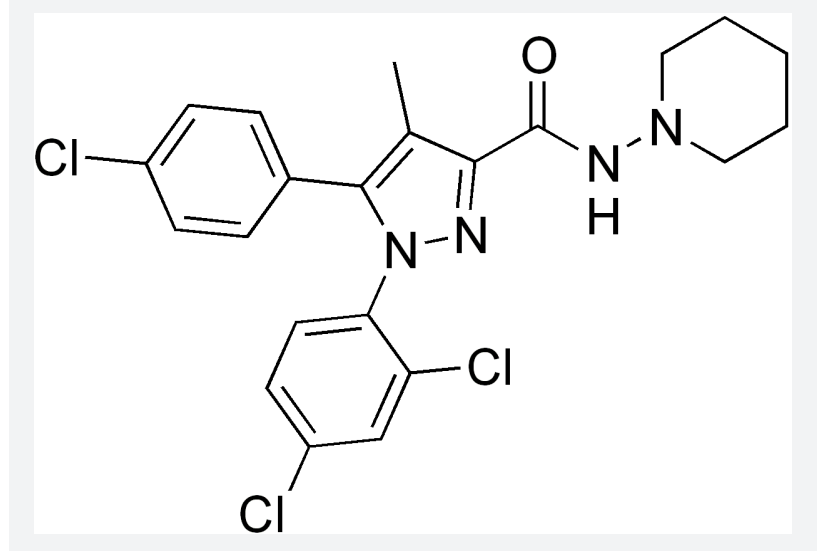


Por otro lado, los cannabinoides inhiben la función motora gástrica y proporcionan protección de la mucosa. Del mismo modo, los agonistas de cannabinoides inhiben el tránsito gastrointestinal ${ }^{12}$.

A nivel endocrino, producen una disminución de la adrenalina y noradrenalina en la médula adrenal que desaparece con la administración repetida de cannabinoides. Los cannabinoides alteran el eje hipotálamo-hipofisario-adrenal (HHA) así como las hormonas responsables del sistema reproductor y la maduración sexual.

A nivel respiratorio se ha descrito un efecto broncodilatador tras el consumo agudo de cannabis. En el consumo crónico por vía respiratoria se observan alteraciones en la función pulmonar tales como un aumento de la prevalencia de bronquitis aguda y crónica, hallazgos endoscópicos de daño de las vías aéreas (eritema, edema, aumento de las secreciones), crecimiento irregular del epitelio bronquial asociado con una expresión alterada de las proteínas que intervienen en la patogénesis del carcinoma de bronquios, anomalías ultraestructurales en los macrófagos alveolares, etc. ${ }^{5}$.

\section{Aplicaciones terapéuticas}

Las investigaciones realizadas en las últimas décadas y el mayor conocimiento de los receptores y sustancias cannabinoides han permitido el desarrollo de fármacos con diferentes indicaciones según sea la interacción con el receptor de tipo antagonista o agonista.

Como se indicó anteriormente, del tipo antagonista o inhibitorio se han desarrollado fármacos como el SR141716A o rimonabant, que inhibe el apetito, de modo que está indicado para personas con problemas de obesidad.

Respecto a los agonistas, el efecto más conocido y por el que se han comercializado derivados cannabinoides en algunos países es el antiemético, como el caso de la nabilona. El tratamiento de las náuseas y vómitos asociados con la quimioterapia fue uno de los primeros usos terapéuticos del cannabis y de los cannabinoides que se evaluaron en pacientes resistentes a otros medicamentos como ondasetrón (antagonista 5- $\mathrm{HT}_{3}$ ) o metoclopramida (antidopaminérgico $\left.\mathrm{D}_{2}\right)^{8}$.

Cabe destacar su efecto analgésico, específicamente en el dolor neuropático, por el cual se ha aprobado el uso en varios países, siendo utilizado en algunas enfermedades como esclerosis múltiple ya que disminuye también la espasticidad, lo cual se detalla más adelante.
Por otra parte, la presencia de ambos receptores CB1 y CB2 en células inmunes y la evidencia de que los cannabinoides inhiben la adenilatociclasa, sugieren un papel en la modulación del sistema inmune. Además, la activación de las células inmunes por una serie de estímulos inflamatorios modulados por la expresión de CB1 y CB2 en estas células, es un hecho que se ha relacionado con los efectos inmunoregulatorios de los cannabinoides.

Se ha investigado que los cannabinoides suprimen la producción de una variedad de citoquinas pro-inflamatorias en cultivos de células humanas y modelos animales, un efecto que se piensa sea mediado principalmente por los receptores CB2. Además, a través de los receptores $C B 2$, los cannabinoides pueden inhibir la producción de factor de necrosis tumoral (TNF)- $\alpha$, e interleucinas por las microglías y los macrófagos. Por otra parte, los receptores CB2 también parecen jugar un papel importante en la regulación de la migración de células de neutrófilos, macrófagos, NK y células B. Se ha sugerido que los cannabinoides son capaces de disminuir la producción de IL- 2 y la proliferación de células $\mathrm{T}$ y puede alterar el perfil de citoquinas de una manera dependiente de CB2. Sin embargo, los receptores CB1 también se han relacionado con la regulación de la neuroinflamación. La anandamida, tiene un papel clave en la transmigración de leucocitos en la esclerosis múltiple. También se demostró que la activación de los receptores CB1 por un agonista sintético selectivo de CB1 (araquidonil-2' cloroetilamida; ACEA) modula condiciones inducidas por el estrés y la neuroinflamación mediante la prevención de la disminución de la captación de glutamato y expresión del transportador de aminoácidos excitatorios 2 (EAAT2), y el aumento de moléculas pro-inflamatorias (citoquinas, factor nuclear kappa B - NF-kB ) y enzimas tales como la sintasa de óxido nítrico inducible (NOS-2) y la ciclooxigenasa-2 (COX-2 ) , además del aumento en la peroxidación de lípidos.

Por lo tanto, debido a la gran variedad de acciones neuroprotectoras, antineuroinflamatorias y antioxidantes, los cannabinoides han sido propuestos como posibles agentes terapéuticos para trastornos neurodegenerativos que combinan las respuestas inflamatorias, como la enfermedad de Alzheimer (EA), la esclerosis múltiple (EM), la esclerosis lateral amioatrófica (ELA), enfermedad de Huntington (EH) y enfermedad de Parkinson (EP), entre otras ${ }^{13}$.

\section{Enfermedades neurodegenerativas}

A continuación se exponen, de forma abreviada, las aplicaciones terapéuticas más importantes de los cannabinoides en las principales enfermedades neurodegenerativas. 


\section{Enfermedad de Alzheimer}

La enfermedad de Alzheimer (EA) es una enfermedad crónica neurodegenerativa debilitante que se asocia con el deterioro cognitivo progresivo y profunda pérdida neuronal.

Una característica de la EA es la presencia de placas seniles compuestas por depósitos extracelulares de patógenos $\beta$-amiloide $(A \beta)$. Se ha propuesto que los fragmentos $A \beta$ juegan un papel central en la génesis de la enfermedad provocando la muerte celular neuronal, donde el hipocampo, corteza cerebral y la amígdala son particularmente más vulnerables. Una segunda característica patológica de la enfermedad es la hiperfosforilación de la proteína asociada a microtúbulos tau, que resulta en la formación de ovillos neurofibrilares intracelulares alterando la comunicación interneuronal. EA también se asocia con eventos neuroinflamatorios y estrés oxidativo que son propensos a exacerbar el proceso de la enfermedad. Las microglías son las células inmunes principales en el cerebro y éstas rodean las placas seniles, posiblemente reclutadas en un intento de eliminar la carga de $A \beta$ por fagocitosis. La deposición $A \beta$ excede la capacidad fagocítica de la microglía y la presencia persistente de microglías activadas conlleva una liberación prolongada de citoquinas pro-inflamatorias y tienen la tendencia a incrementar el procesamiento de la proteína precursora de amiloide para generar más fragmentos $A \beta$, así como tener una influencia directa neurotóxica. La asociación de las microglías activadas en la periferia de las placas seniles contribuye a la generación de especies reactivas de oxígeno que median el daño oxidativo que se encuentra en los cerebros de pacientes con EA. Otros factores que se cree que contribuyen a la fisiopatología de la EA incluyen la desregulación de la homeostasis del calcio intracelular y la citotoxicidad. Actualmente no hay tratamientos disponibles para revertir la progresión de la enfermedad.

El antagonista del receptor NMDA no competitivo, la memantina, se utiliza en el tratamiento de moderado a grave en la EA, y sus propiedades beneficiosas se basan en una capacidad para inhibir patológica, pero no fisiológicamente, funciones de los receptores de NMDA, así como la acción antioxidante y una predisposición a aumentar la producción de factor neurotrófico en el cerebro. Los efectos protectores de algunos cannabinoides están relacionados con la regulación directa del receptor de NMDA, ya que los cannabinoides no psicotrópicos, como HU-211, actúan como un inhibidor estereoselectivo del receptor de $\mathrm{NMDA}^{14}$.

Además, la activación de los receptores CB1 protege las neuronas de la citotoxicidad, y también inhiben la liberación de glutamato, lo que puede contribuir a una reducción de esta citotoxicidad.

Los cannabinoides pueden reducir el estrés oxidativo, la neuroinflamación y la apoptosis que es provocada por $\mathrm{A} \beta$, promoviendo mecanismos de reparación intrínsecos del cerebro. Algunos cannabinoides, como el $\Delta^{9}$-THC, también pueden aumentar la disponibilidad y reducir ACh amiloidogénesis, aunque los posibles efectos secundarios psicoactivos pueden obstaculizar su utilidad clínica ${ }^{15}$.

Es de especial interés el receptor CB2 para reducir la neuroinflamación y la estimulación de la neurogénesis, dada la reducción del riesgo de la actividad psicotrópica y la estrecha asociación de los receptores CB2 en la placa senil, lo que limita los efectos del fármaco en la región de la patología y la preservación del posibilidad de efectos generalizados sobre los procesos neurofisiológicos normales ${ }^{14}$ (Figura 7).

Figura 7. Posibles sitios de acción terapéutica de los cannabinoides en la enfermedad de Alzheimer
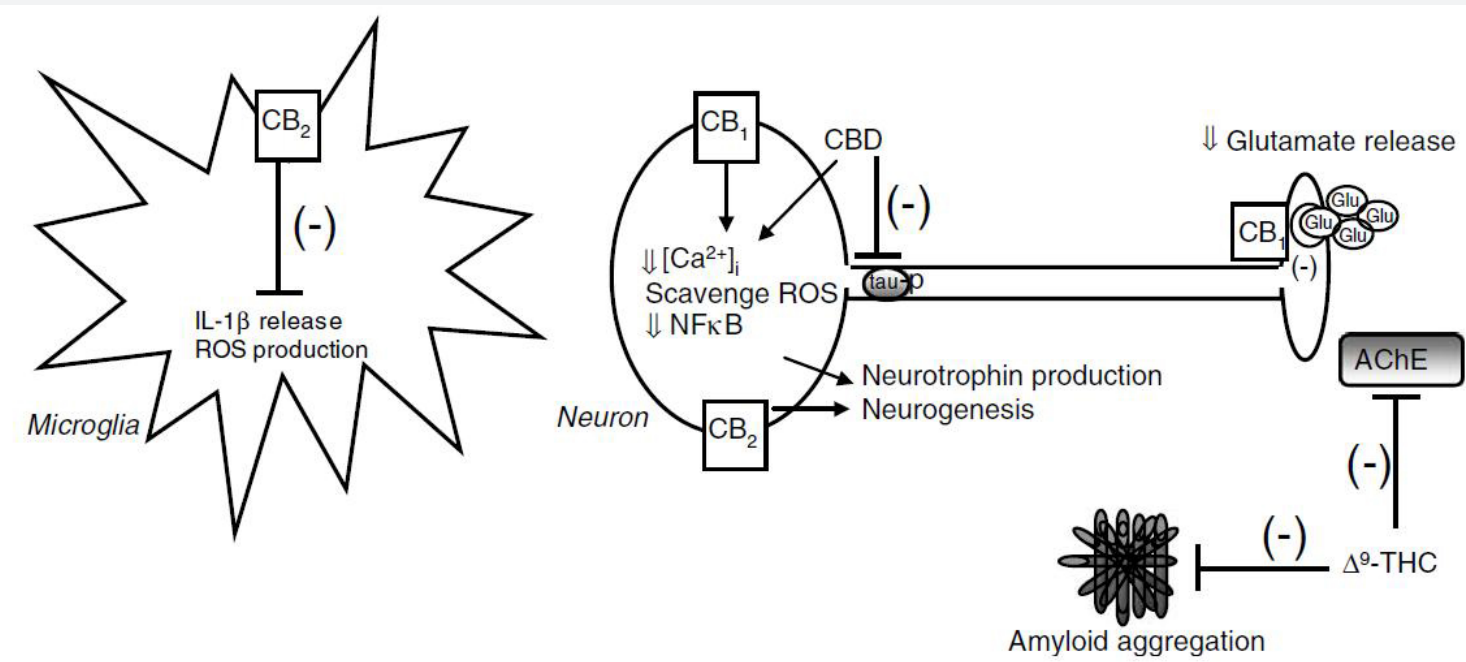


\section{Enfermedad de Huntington}

La enfermedad de Huntington (EH) es un trastorno neurodegenerativo hereditario caracterizado por anormalidades motoras, disfunción cognitiva y síntomas psiquiátricos. La causa principal de la enfermedad es una mutación en el gen de la huntingtina que consiste en una expansión de repetición de triplete CAG traducido en una poliglutamina anormal en la porción amino-terminal de esta proteína que se convierte tóxica (Figura 8). Los primeros síntomas de la $\mathrm{EH}$ pueden incluir movimientos descontrolados, torpeza, y problemas de equilibrio. Más tarde, la enfermedad de Huntington puede inhabilitar la capacidad de caminar, hablar y tragar. Algunas personas dejan de reconocer a sus familiares. En la actualidad, no existe farmacoterapia específica para aliviar los síntomas motores y cognitivos y/o para detener/ retrasar la progresión de la enfermedad en EH.
Los cannabinoides con perfil antioxidante, es decir, $\Delta^{9}$-THC y CBD, protegen las neuronas estriales contra la toxicidad causada por el complejo mitocondrial II inhibidor ácido 3-nitropropiónico (3NP) que produce la lesión oxidativa. Alivian los síntomas hipercinéticos, dado sus efectos inhibitorios sobre el movimiento $y$, en particular, se están estudiando como agentes modificadores de la enfermedad debido a sus propiedades antiinflamatorias, neuroprotectoras y neuroregenerativas ${ }^{16}$. Concretamente, dado que los resultados con los receptores CB1 no son satisfactorios, se están empleado agonistas y antagonistas específicos de los receptores CB2 ya que han demostrado mejores resultados aunque están aún en pruebas poco concluyentes.

Por otra parte, la activación de los receptores cannabinoides puede inhibir la liberación de GABA, que aumenta en la mayoría de enfermos de Huntington ${ }^{5}$.

Figura 8. Proceso de apoptosis en una neurona con Huntington

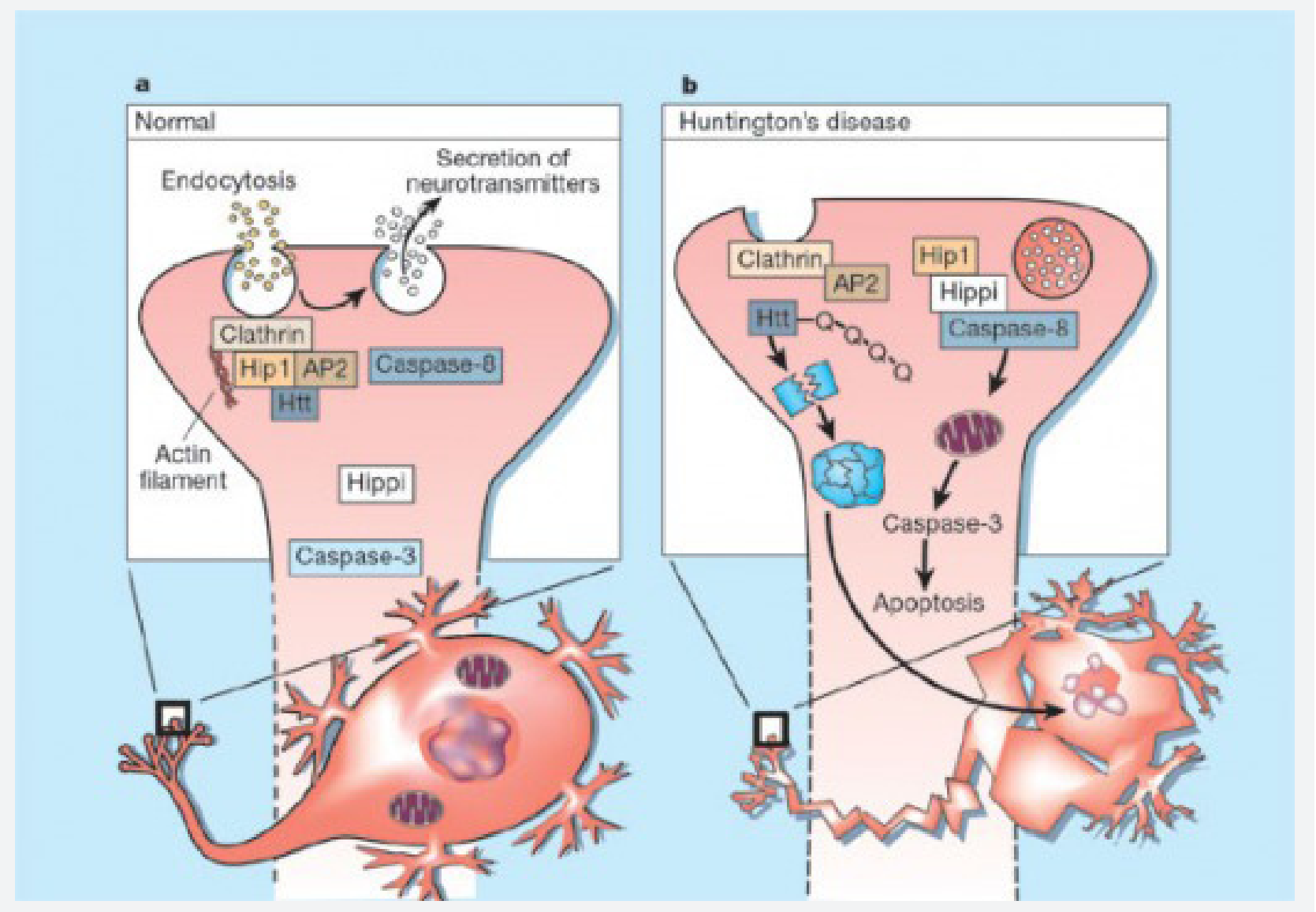

\section{Enfermedad de Parkinson}

La enfermedad de Parkinson (EP) es un trastorno neurodegenerativo progresivo. Los principales síntomas clínicos en la EP son temblor, bradiquinesia, inestabilidad postural y rigidez, síntomas que resultan de la denervación dopami- nérgica severa del cuerpo estriado causada por la muerte progresiva de las neuronas dopaminérgicas de la sustancia negra (Figura 9). Los principales síntomas de la EP se pueden atenuar con la terapia de reemplazo dopaminérgico. Sin embargo, este tratamiento no funciona para todos los pacientes con EP, y cuando se utiliza durante más de 5-10 
años, provoca un estado discinético irreversible. Por lo tanto, la búsqueda de nuevas terapias sintomáticas, así como tratamientos eficaces en el retraso de la progresión del daño nigroestriatal, es todavía el principal reto en la terapia de la $\mathrm{EP}^{6}$.

Algunos compuestos a base de cannabinoides han sido recientemente propuestos como terapias prometedoras en la EP. El bloqueo de los receptores CB1, que son muy abundantes en las estructuras de los ganglios basales, puede ser eficaz en la reducción de la inhibición típica motora de los pacientes con $\mathrm{EP}^{9}$. Sin embargo, la eficacia del bloqueo de los receptores CB1 se restringió a circunstancias específicas, que es el uso de dosis bajas y daños muy extensos en la sustancia negra.

Se ha comprobado que algunos cannabinoides actúan como protectores de las neuronas de la sustancia negra contra la muerte causada por diferentes estímulos citotóxicos en varios modelos experimentales de EP. Estos incluyen $\Delta^{9}$-THC y CBD, el agonista del receptor CB1/CB2 sintético CP55940 y el análogo de anandamida AM404. Estos compuestos actúan a través de mecanismos antioxidantes que parecen ser independientes de los receptores CB1 o CB2. También cabe destacar que ratones deficientes en el receptor CB1 muestran una mayor vulnerabilidad a las lesiones de 6-hidroxidopamina. Sin embargo, los agonistas selectivos de dicho receptor no parecen proteger contra los daños de la 6-hidroxidopamina y pueden incluso agravar principales síntomas parkinsonianos, dado que los efectos hipocinéticos están asociados con la activación de los receptores CB1.

Por lo tanto, los datos anteriores proporcionan una buena evidencia de que un cannabinoide con propiedades antioxidantes y capacidad de activar los receptores CB2, pero bloqueando a su vez los receptores CB1, podría servir para aliviar los síntomas parkinsonianos y para detener/retardar de la neurodegeneración en la EP.

El fitocannabinoide $\Delta^{9}$-tetrahidrocannabivarina $\left(\Delta^{9}-\mathrm{THCV}\right)$ tiene un perfil farmacológico que parece ser particularmente apropiado para la $\mathrm{EP}^{17}$.

Figura 9. Imagen de la muerte de las células dopaminérgicas (desaparición del color rojo) en la sustancia negra

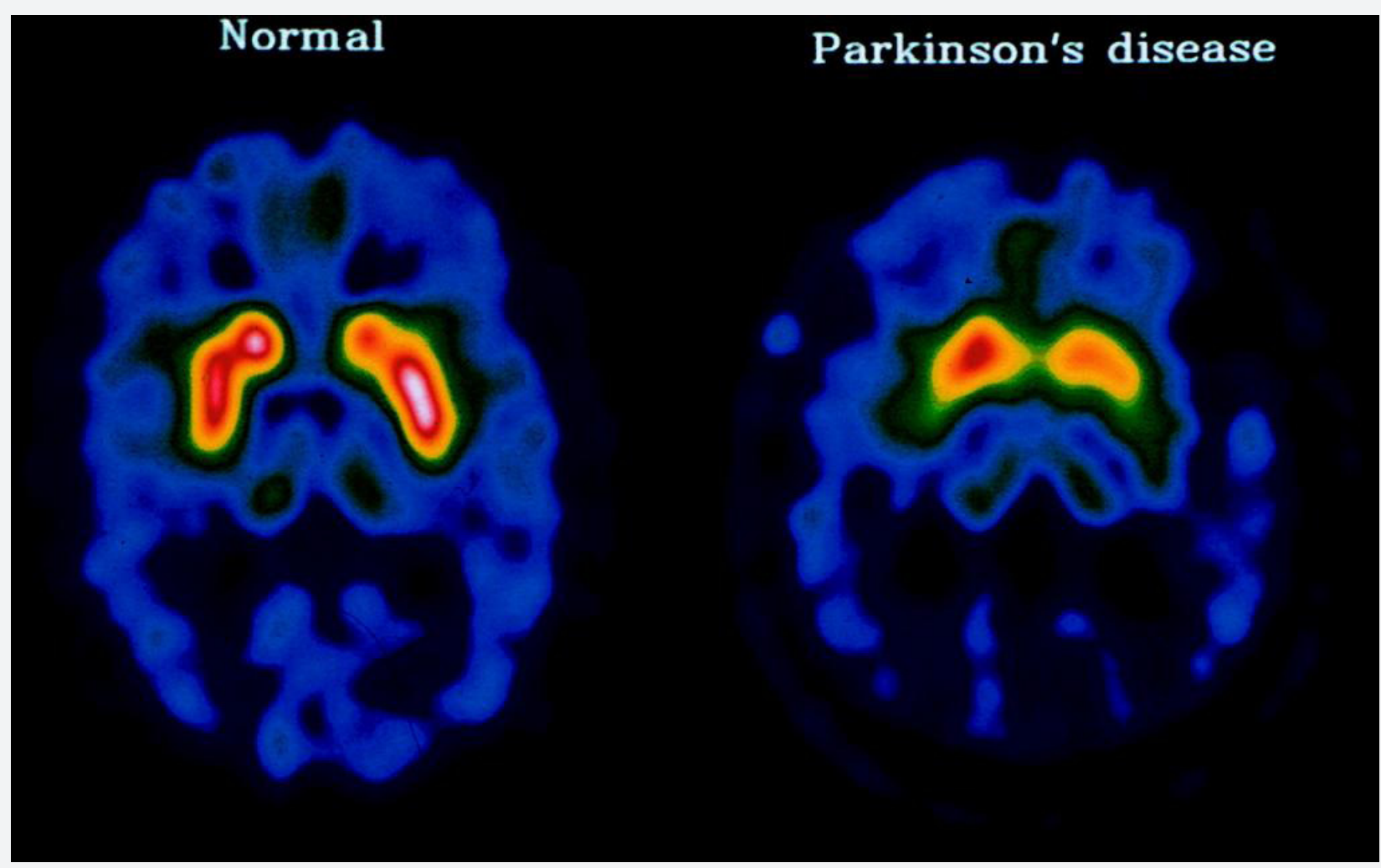




\section{Enfermedad de Esclerosis Múltiple}

La esclerosis múltiple (EM) es una enfermedad crónica que afecta al SNC, con una fase inflamatoria y otra fase neurodegenerativa. Suele aparecer en personas jóvenes y causa grave discapacidad física y mental así como un deterioro importante de la calidad de vida. Morfológicamente la EM se caracteriza por inflamación, desmielinización y daño axonal.

Los pacientes con EM tienen síntomas muy variados: alteraciones de la sensibilidad, paresias, temblor, diplopía, visión borrosa, alteraciones de los esfínteres, fatiga, alteraciones de la marcha, etc. La espasticidad es uno de los síntomas más frecuentes e invalidantes asociados a la EM ${ }^{18}$

Aunque la espasticidad es potencialmente tratable, revisiones sistemáticas demuestran que la efectividad de los medicamentos indicados es limitada y que sólo proporcionan un beneficio clínico modesto, con algunos pacientes que no responden al tratamiento. Las opciones para los pacientes con espasticidad refractaria al tratamiento es limitado, costoso e invasivo (por ejemplo baclofeno).

Gracias a la interacción que tiene las sustancias cannabinoides con los receptores cannabinoides y éstos con los neurotransmisores, especialmente con el GABA, se ha demostrado que asociaciones de $\Delta^{9}$-THC y CBD disminuyen los movimientos espásticos en personas con EM e incluso proporciona efectos analgésicos.
Estudios realizados con placebo afirman que la administración de $\Delta^{9}$-THC y CBD en conjunto (ya que mejoran la tolerancia y efectividad) mejoran la rigidez apenas $10 \mathrm{~min}$ después de la administración intravenosa, y que incluso altas dosis tienen la misma eficacia que otros medicamentos como el baclofeno.

En comparación con el placebo, la asociación $\Delta^{9}$-THC/CBD y el $\Delta^{9}$-THC mejoran significativamente el espasmo muscular y la espasticidad, e incluso la severa (Figura 10) ${ }^{19}$.

Sativex ${ }^{\circledR}$ (asociación $\Delta^{9}$-THC/CBD) parece constituir un avance clave en este campo. Su formulación para pulverización bucal presenta claras ventajas farmacocinéticas respecto al consumo tradicional inhalatorio con el empleo de cannabis, ya que la combinación 1:1 de $\Delta^{9}$-THC y CBD parece reducir drásticamente el riesgo de que se produzcan los efectos psicotrópicos característicos del consumo de cannabis $^{18}$.

Por otra parte, existen evidencias de que el uso de las sustancias cannabinoides pueden ejercer un efecto neuroprotector en enfermos de EM. Sin embargo, los estudios realizados no muestran una respuesta positiva al respecto. No obstante, se sigue investigando este efecto, ya que los resultados pueden haber sido incorrectos debido a la duración y uso de otros medicamentos durante los estudios ${ }^{20}$.

Figura 10. Efecto de la asociación $\triangle 9$-THC/CDB sobre la espasticidad intratable y espasmos musculares en pacientes con diversas enfermedades neurológicas, con base a la valoración del paciente (VP) $(100=$ mejor puntuación), y Valoraciones de observadores $(\mathrm{VO})(10=$ peor puntuación) de la espasticidad severa. Ambos estudios durante dos semanas de tratamiento
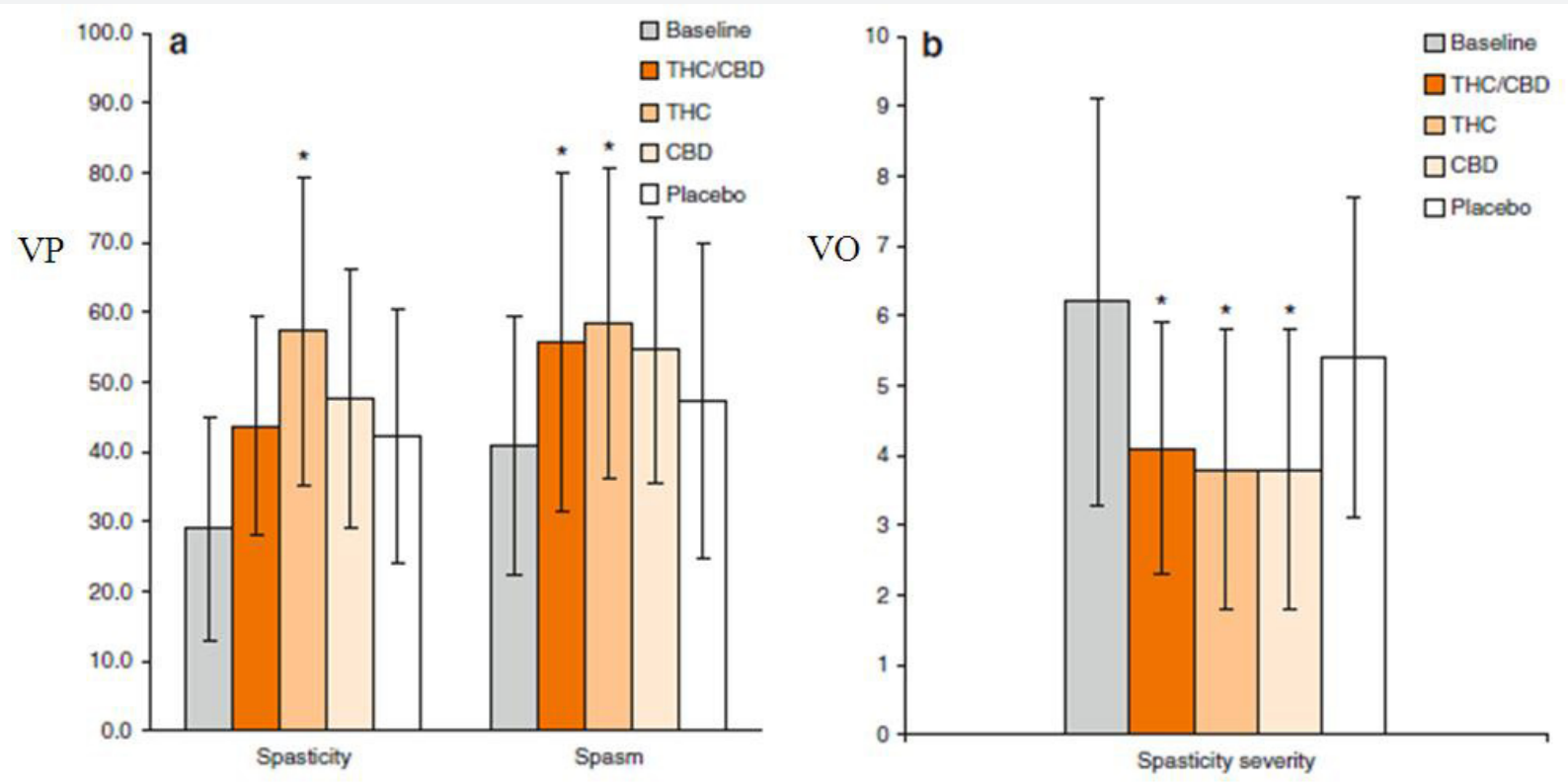


\section{Enfermedad de esclerosis lateral amiotrófica}

La esclerosis lateral amiotrófica es la forma más común de enfermedad en las neuronas motoras del adulto. Es una enfermedad neuromuscular rápidamente progresiva y que destruye las neuronas motoras superiores e inferiores, provocando debilidad, espasticidad, y finalmente la muerte por insuficiencia respiratoria. La gran mayoría de los casos de ELA se adquieren y se producen esporádicamente.

Nuevas evidencias sugieren que el aumento del estrés oxidativo, la toxicidad de los radicales libres y/o la actividad excesiva de glutamato es lo que conduce a la muerte celular de las neuronas motoras en el cerebro y médula espinal ${ }^{6}$. Se ha establecido que la ELA también involucra otras células no neuronales incluyendo astroglía y microglía. Otros posibles mecanismos implicados en la degeneración de las neuronas motoras en la ELA incluyen disfunción mitocondrial, neuroinflamación, deficiencia de factor de crecimiento y excitotoxicidad del glutamato. A este respecto, el único tratamiento aprobado por la FDA es el Riluzol, a pesar de su limitada eficacia terapéutica.

Debido a los efectos de los derivados cannabinoides en las moléculas pro-inflamatorias y excitotóxicas, se han comenzado numerosos estudios respecto al posible efecto neuroprotector en esta enfermedad, aunque todavía no han dado resultados claros. Se ha comprobado que mejora los síntomas incluyendo el alivio del dolor y los espasmos musculares, mejora el apetito, disminuye la depresión y ayuda a gestionar la sialorrea (babeo excesivo) al secar la saliva en la boca ${ }^{21}$.

\section{CONCLUSIONES}

En los últimos años se ha demostrado el gran potencial de las sustancias cannabinoides en aplicaciones terapéuticas más allá de su uso analgésico o antiemético, esto es, en enfermedades neurodegenerativas en las que pueden no solo disminuir los síntomas, sino frenar el proceso de la enfermedad. Además la posible aplicación como sustancias anticancerosas abre aún más puertas a su uso, siendo particularmente notable la creciente actividad investigadora realizada en los últimos 15 años.

Hasta ahora y a pesar de sus demostrados efectos beneficiosos derivados del uso de cannabinoides, cabe destacar sus efectos adversos y su efectividad parcial en algunas enfermedades, poniendo en controversia su uso y autorización de medicamentos con estos principios activos. Investigaciones recientes afirman, que los efectos secundarios provocados por los derivados cannabinoides son producidos por la interacción de éstos con el receptor CB1, no teniendo apenas efectos secundarios la interacción con el CB2.
Además, los últimos ensayos clínicos demuestran que en la mayoría de las enfermedades neurodegenerativas como Alzheimer, Parkinson y Huntington, la interacción con el receptor CB2 de forma exclusiva es la que da una respuesta positiva en la neuroprotección.

\section{REFERENCIAS}

1. Bruneton J, Villar del Fresno AM, Carretero Accame E, Rebuelta Lizabe M. Farmacognosia: fitoquímica, plantas medicinales. $2^{\text {a }}$ ed. Zaragoza: Editorial Acribia; 2001. 1099 p.

2. Bravo Díaz L. Farmacognosia. Madrid: Editorial Elsevier; 2003. $356 \mathrm{p}$.

3. Abood ME, Sorensen RG, Stella N. endoCANNABINOIDS. New York: Editorial Springer; 2013. 286 p.

4. Niesink RJ, van Laar MW. Does Cannabidiol Protect Against Adverse Psychological Effects of THC? Front. Psychiatry. 2013; 16 (4): 130 (doi: 10.3389/fpsyt.2013.00130)

5. Muñoz-Rubio I, Cózar-Bernal M.J, Álvarez-Fuentes J, MartínBanderas L, Fernández-Arévalo M, Holgado M.A. Aplicaciones de los cannabinoides como agentes terapéuticos. Ind. Farm. 2011; 163: 68-77

6. Köfalvi A. Cannabinoids and the Brain. New York: Editorial Springer; 2008. $583 \mathrm{p}$.

7. Stella N. Chronic THC intake modifies fundamental cerebellar functions. J. Clin. Invest. 2013; 123 (8): 3208-3210.

8. Zuardi AW. Cannabidiol: from an inactive cannabinoid to a drug with wide spectrum of action. Rev. Bras. Psiquiatr. 2008; 30 (3): 271-280.

9. Reggio PH. The Cannabinoid Receptors. Greensboro: Editorial Springer; 2009. $402 \mathrm{p}$.

10. Hernán Perez de la Ossa, D, Lorente M, Gil-Alegre ME, Torres S, Garcia-Taboada E, Aberturas M.R. Local delivery of cannabinoid-loaded microparticles inhibits tumor growth in a murine xenograft model of glioblastoma multiforme. PLoS One 2013; 8 (1): e54795.

11. Molina PE, Amedee A, LeCapitaine NJ, Zabaleta J, Mohan M, Winsauer P, Cannabinoid neuroimmune modulation of SIV disease. J. Neuroimmune Pharmacol. 2011; 6 (4): 516-527.

12. Krowicki ZK. Involvement of hindbrain and peripheral prostanoids in gastric motor and cardiovascular responses to delta-9-tetrahydrocannabinol in the rat. J. Physiol. Pharmacol. 2012; 63 (6): 581-588.

13. Saito VM, Rezende RM, Teixeira AL. Cannabinoid modulation of neuroinflammatory disorders. Curr. Neuropharmacol. 2012; 10 (2): 159-166.

14. Campbell VA, Gowran A. Alzheimer's disease; taking the edge off with cannabinoids? Br. J. Pharmacol. 2007; 152 (5): 655-662. 
15. Martin-Moreno AM, Brera B, Spuch C, Carro E, Garcia-Garcia L, Delgado M, Pozo MA, Innamorato NG, Cuadrado A, de Ceballos ML. Prolonged oral cannabinoid administration prevents neuroinflammation, lowers beta-amyloid levels and improves cognitive performance in Tg APP 2576 mice. J Neuroinflammation 2012; 9 (8): 1-15

16. Valdeolivas S, Satta V, Pertwee RG, Fernandez-Ruiz J, Sagredo O. Sativex-like combination of phytocannabinoids is neuroprotective in malonate-lesioned rats, an inflammatory model of Huntington's disease: role of CB1 and CB2 receptors. ACS Chem. Neurosci. 2012; 3 (5): 400-406.

17. Garcia C, Palomo-Garo C, Garcia-Arencibia M, Ramos J, Pertwee R, Fernandez-Ruiz J. Symptom-relieving and neuroprotective effects of the phytocannabinoid Delta(9)-THCV in animal models of Parkinson's disease. Br. J. Pharmacol. 2011; 163 (7): 1495-1506
18. Oreja-Guevara C. Treatment of spasticity in multiple sclerosis: new perspectives regarding the use of cannabinoids. Rev. Neurol. 2012; 55 (7): 421-430.

19. Syed YY, McKeage K, Scott LJ. Delta-9-tetrahydrocannabinol/cannabidiol (Sativex®): a review of its use in patients with moderate to severe spasticity due to multiple sclerosis. Drugs 2014; 74 (5): 563-578.

20. Zajicek J, Ball S, Wright D, Vickery J, Nunn A, Miller D, Effect of dronabinol on progression in progressive multiple sclerosis (CUPID): a randomised, placebo-controlled trial. Lancet Neurol. 2013; 12 (9): 857-865.

21. Carter GT, Abood ME, Aggarwal SK, Weiss MD. Cannabis and amyotrophic lateral sclerosis: hypothetical and practical applications, and a call for clinical trials. Am. J. Hosp. Palliat. Care 2010; 27 (5): 347-356. 